\title{
A diferenciação cognitiva na infância: um estudo de perfis cognitivos aos 5, 7 e 9 anos
}

\author{
Cognitive differentiation during childhood: A study \\ on cognitive profiles of 5, 7, and 9-year-old children
}

\author{
Ana Azevedo MARTINS \\ Diana Lopes SOARES \\ Lurdes BRITO' \\ Gina Cláudia LEMOS \\ Ana Filipa ALVES ${ }^{1}$ \\ Leandro da Silva ALMEIDA ${ }^{1}$
}

\section{Resumo}

No seio do debate sobre se a inteligência é mais bem definida por um fator geral ou por aptidões específicas, ganha relevância a hipótese da diferenciação cognitiva. Análises recentes enfatizam o interesse dessa questão para a investigação e alertam para a relevância das suas implicações na área educativa. Este estudo analisou a possibilidade de a diferenciação das aptidões cognitivas ocorrer já na infância e também o efeito moderador do Quociente de Inteligência na magnitude da relação entre as habilidades cognitivas. Aplicou-se uma bateria de provas que avaliam várias funções cognitivas a uma amostra de 231 crianças com 5, 7 e 9 anos, distribuídas por três grupos de desempenho cognitivo. Os resultados de uma análise de clusters hierárquica e de uma análise de variância apontam para a não diferenciação das funções cognitivas na infância. Contudo, uma análise mais cuidadosa aponta para alguma diferenciação suportada pela heterogeneidade dos perfis cognitivos junto dos alunos com Quociente de Inteligência elevado.

Palavras-chave: Aptidão cognitiva; Infância; Inteligência; Quociente de inteligência.

\begin{abstract}
Within the debate about whether intelligence is best defined by a general factor or specific skills, the hypothesis of cognitive differentiation gains relevance. Recent analyses have emphasized the importance of this issue in the investigation of cognitive skills and have highlighted its implications in education. This study examined the possibility that the differentiation of cognitive abilities may occur during childhood and investigated the moderating effect of Intelligence Quotient on the magnitude of the relationship between cognitive abilities. A battery of tests for assessing

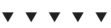

1 Universidade do Minho, Centro de Investigação em Educação. Campus de Gualtar, 4710-057, Braga, Portugal. Correspondência para/ Correspondence to: A.A. MARTINS. E-mail: <anaazevedo.martins@gmail.com>.

Apoio: Fundação para a Ciência e a Tecnologia (Projeto SFRH/BD/84153/2012).
\end{abstract}


cognitive function was administered to 231 children aged 5, 7, and 9 years old, who were divided into three cognitive performance groups. The results of hierarchical cluster analysis and variance analysis indicate the lack of differentiation of cognitive functions during childhood. However, a more careful analysis suggests some differentiation supported by the heterogeneity of cognitive profiles among students with high Intelligence Quotient.

Keywords: Cognitive ability; Childhood; Intelligence; Intelligence quotient.

É difícil definir a inteligência de forma unânime, pois são diferentes os modelos teóricos presentes. Atualmente, algum consenso foi obtido a partir de uma organização estratificada das habilidades cognitivas. Considerando o modelo de Cattell-Horn-Carroll (Teoria CHC), pode-se assumir que a inteligência se organize em três estratos (Almeida, 2002; Primi, 2002): o primeiro formado por algumas dezenas de habilidades específicas de primeira ordem e próximas do desempenho de tarefas singulares; o segundo reunindo uma dezena de fatores mais gerais de segunda ordem, traduzindo processos cognitivos (codificação, memória, raciocínio etc.) ou conteúdos de tarefas (quantitativo, verbal, figurativo etc.); e, por último, o fator geral de terceira ordem, próximo do fator $g$ de Spearman (1927).

A par da controvérsia em torno do número dos fatores que definem a inteligência, alguma dissonância persiste quanto à sua diferenciação conforme os níveis de desempenho cognitivo ou conforme o desenvolvimento ao longo da idade. No primeiro caso, e associado ao fator $g$, Spearman (1927) postula "the law of diminishing returns" (SLODR, Spearman's Law of Diminishing Returns), segundo a qual as correlações entre os testes de desempenho cognitivo e os respectivos loadings em $g$ diminuiriam para indivíduos com elevado Quociente de Inteligência (QI). Essa relação inversa entre o QI e a magnitude da correlação dos testes tem encontrado suporte empírico em vários estudos (Carlstedt, 2001; Coyle \& Rindermann, 2013; Jensen, 2003). Facon (2006) analisou a magnitude das correlações entre os resultados nos subtestes da Wechsler Intelligence Scale for Children, Third Edition (WISC-III) por referência à distribuição do nível de habilidade cognitiva (alto e baixo QI) de três grupos etários definidos (7-9 anos, 10-12 anos e 13-15 anos). Verificou que a magnitude dessas correlações é praticamente igual nos grupos de alto e baixo QI até a faixa etária dos 10-12 anos. A partir daí, e como seria previsto pela SLODR, as correlações tornam-se mais fortes para os indivíduos com baixo QI. Num estudo anterior com crianças de 4 e 9 anos, Facon (2004) também não teria encontrado evidências para a diferenciação cognitiva, tendo assumido que esse fenômeno emergia, apenas, em idades mais avançadas (Facon, 2006).

Uma segunda abordagem associa a diferenciação das habilidades cognitivas às experiências educacionais e interesses dos sujeitos, sobretudo a partir da adolescência. Nessa linha, Cattell (1987) aprofundou o conceito do fator $g$ e subdividiu-o em inteligência fluida ( $g f$ ) e inteligência cristalizada (gc), assumindo a primeira como mais marcada por aspetos genéticos e neurológicos, enquanto a segunda decorre das experiências culturais e educacionais (Kan, Kievit, Dolan, \& van der Maas, 2011; Primi, Ferrão, \& Almeida, 2010). Aprofundando a relação entre as duas inteligências, Cattell (1987) propõe a teoria do investimento e assume que o desenvolvimento da capacidade geral ( $g f$ ) até ao final da adolescência seria acompanhado por um investimento progressivo em áreas delimitadas do conhecimento ou da realização, decorrendo daí um amplo conjunto de capacidades cristalizadas ( $g c$ ). Assim, à medida que o indivíduo avança no seu percurso desenvolvimental, observa-se uma crescente especialização das habilidades cognitivas e consequente redução de variância explicada por $g$ (Juan-Espinosa et al., 2002).

Apesar de os resultados de alguns estudos não sustentarem a hipótese da diferenciação cognitiva (Juan-Espinosa, Cuevas, Escorial, \& García, 2006; Molenaar, Dolan, Wicherts, \& van der Maas, 2010; Rietveld, Dolan, van Baal, \& Boomsma, 2003), existem outros que apoiam esse fenômeno e a possibilidade da sua ocorrência mesmo antes da adolescência (Tideman \& Gustafsson, 2004; Tusing \& Ford, 2004). 
Assim, no presente estudo, considerando-se os resultados de crianças com 5, 7 e 9 anos, numa bateria que avalia vários processos cognitivos em provas verbais e não-verbais, pretende-se verificar se uma eventual diferenciação das habilidades cognitivas ocorre já na infância. Em segundo lugar, e tendo em conta os três grupos etários, pretende-se analisar que perfis cognitivos emergem em função dos resultados obtidos.

\section{Método}

\section{Participantes}

Participaram no presente estudo 231 crianças de 5,7 e 9 anos $(M=7,50 ; \pm 3,31)$, que frequentavam a pré-escola e o primeiro ciclo do ensino fundamental, em instituições públicas e privadas da zona norte de Portugal. A amostra foi estratificada em função do sexo e da comunidade de pertença (meio urbano/meio rural). Crianças sinalizadas com necessidades educativas especiais não foram consideradas.

\section{Instrumentos}

A Escala de Competências Cognitivas para Crianças dos 4 aos 10 anos - ECCOs 4/10 (Brito \& Almeida, 2009) é uma bateria de aplicação individual, composta por 11 provas que avaliam seis processos cognitivos (perceção, memória de curto prazo, compreensão, raciocínio, resolução de problemas e pensamento divergente), recorrendo a tarefas de conteúdo verbal e não-verbal. Neste estudo não se utilizou a prova que avalia o processo perceptivo, em virtude de não haver uma prova paralela recorrendo ao conteúdo verbal. Estudos de precisão e de validade dos resultados na bateria apontam índices de consistência interna elevados, compreendidos entre 0,87 e 0,97. Quanto à validade de constructo, os autores defendem um fator geral saturando todas as provas, exceto as relativas ao pensamento divergente (Brito \& Almeida, 2009).

\section{Procedimentos}

A bateria foi aplicada individualmente, em duas sessões separadas, com duração entre 60 e 90 minutos. Os professores e os profissionais de educação foram esclarecidos da natureza, objetivos do estudo e condições de realização, assim como dos cuidados éticos respeitados (anonimato, confidencialidade, proteção e segurança dos dados recolhidos). A bateria foi aplicada por psicólogos previamente treinados.

Os dados foram analisados através do software IBM Statistical Package for the Social Sciences (versão 22.0 para Windows). Calcularam-se as notas estandardizadas, convertendo-se os resultados obtidos numa escala de 1 a 20 pontos (média de 10 e desvio-padrão de 3 pontos, assumido esse desvio como critério para a diferenciação cognitiva). Realizou-se uma análise de clusters, seguindo os passos de Bergman (1998), começando com o estudo da correlação entre as variáveis e sua eventual multicolinearidade. Para a análise de clusters recorreu-se ao hierarquical clustering algorithm (Everitt, Landau, \& Leese, 2001) e ao método Ward. A identificação dos clusters seguiu os três passos usuais: análise do dendograma e dos coeficientes de aglomeração; interpretação teórica de cada cluster e análise descritiva das variáveis que o compõe; análise de variância ( $F$ da Anova, Analysis of Variance) e identificação das variáveis que significativamente diferenciam os grupos (teste de contraste).

\section{Resultados}

Na Tabela 1 apresentam-se as estatísticas descritivas e os coeficientes de correlação dos resultados nas provas da ECCOs 4/10. As médias nas provas assumem posições intermédias em comparação aos valores mínimo e máximo da distribuição. Os coeficientes de assimetria e curtose aproximam-se de zero, estando em sintonia com uma distribuição gaussiana dos resultados em cada prova. Os índices de correlação variam entre 0,13 $(p=0,04)$ e $0,53(p=0,00)$, respeitando o pressuposto da ausência de multicolinearidade, necessário à prossecução das análises posteriores. 
Tabela 1

Estatísticas descritivas e coeficientes de correlação. Porto, 2013

\begin{tabular}{|c|c|c|c|c|c|c|c|c|c|c|}
\hline Variáveis & MNV & CNV & RNV & RPNV & PDNV & $\mathrm{MV}$ & $\mathrm{CV}$ & RV & RPV & PDV \\
\hline MNV & - & & & & & & & & & \\
\hline CNV & $0,19^{* \star}$ & - & & & & & & & & \\
\hline RNV & $0,39^{* * *}$ & $0,31^{* * *}$ & - & & & & & & & \\
\hline RPNV & $0,23^{* \star *}$ & $0,28^{* * *}$ & $0,33^{* * *}$ & - & & & & & & \\
\hline PDNV & 0,06 & $0,18^{* *}$ & 0,02 & $0,21^{* *}$ & - & & & & & \\
\hline MV & $0,13^{*}$ & $0,39^{* \star *}$ & $0,37^{* * *}$ & $0,28^{* * *}$ & 0,12 & - & & & & \\
\hline$C V$ & $0,23^{* * *}$ & $0,43^{* \star *}$ & $0,38^{* * *}$ & $0,35^{* * *}$ & $0,26^{* * *}$ & $0,42^{* * *}$ & - & & & \\
\hline RV & $0,14^{*}$ & $0,42^{* * *}$ & $0,35^{* * *}$ & $0,26^{* * *}$ & 0,11 & $0,53^{* * *}$ & $0,50^{* * *}$ & - & & \\
\hline RPV & $0,33^{* * *}$ & $0,37^{* * *}$ & $0,42^{* * *}$ & $0,38^{* * *}$ & $0,14^{*}$ & $0,37^{* * *}$ & $0,48^{* * *}$ & $0,45^{* * *}$ & - & \\
\hline PDV & 0,11 & 0,12 & 0,07 & 0,02 & $0,26^{* * *}$ & $-0,00$ & $0,24^{* * *}$ & 0,13 & 0,08 & - \\
\hline N & 231 & 231 & 231 & 231 & 231 & 231 & 231 & 231 & 231 & 231 \\
\hline Min-Max & 0-19 & $1-19$ & $1-19$ & $1-19$ & 0-19 & $1-18$ & $1-19$ & $1-18$ & $1-19$ & $0-19$ \\
\hline$M$ & 9,65 & 9,93 & 9,21 & 9,68 & 9,22 & 9,44 & 9,43 & 9,62 & 10,00 & 9,26 \\
\hline$D P$ & 3,36 & 3,16 & 3,70 & 3,55 & 3,18 & 3,33 & 3,83 & 3,35 & 3,34 & 3,77 \\
\hline Sk & $-0,16$ & $-0,14$ & $-0,05$ & 0,10 & $-0,02$ & $-0,47$ & $-0,34$ & $-0,40$ & 0,16 & $-0,06$ \\
\hline Ku & 0,38 & 0,44 & 0,01 & 0,10 & 0,38 & 0,54 & $-0,34$ & 0,36 & 0,19 & 0,38 \\
\hline
\end{tabular}

Nota: ${ }^{*} p<0,05 ;{ }^{* *} p<0,01 ;{ }^{* * *} p<0,001$

MNV: Memória Não-Verbal; CNV: Compreensão Não-Verbal; RNV: Raciocínio Não-Verbal; RPNV: Resolução de Problemas Não-Verbal; PDNV: Pensamento Divergente Não-Verbal; MV: Memória Verbal; CV: Compreensão Verbal; RV: Raciocínio Verbal; RPV: Resolução de Problemas Verbal; PDV: Pensamento Divergente Verbal; N: Dimensão da amostra; Min-Max: Mínimo-Máximo; M: Média; DP: Desvio-Padrão; Sk: Assimetria; Ku: Curtose.

Através da análise hierárquica de clusters e do respetivo dendograma, foi gerada uma solução final com três grupos distintos, apresentada na Tabela 2. Os grupos organizam-se segundo os níveis gerais de desempenho na ECCOs 4/10 e da seguinte forma: Grupo 1, alunos com alto desempenho cognitivo (QI alto); Grupo 2, alunos com médio desempenho cognitivo (QI médio); Grupo 3, alunos com baixo desempenho cognitivo (QI baixo). Como seria de antecipar, as médias nas várias provas diminuíram progressivamente do Grupo 1 para o Grupo 2, e do Grupo 2 para o Grupo 3. A única exceção observa-se nas provas de pensamento divergente de ambos os conteúdos (verbal e não-verbal), em que a média do Grupo 2 suplanta a média observada no Grupo 1 (diferença de 0,36 e 0,86, respectivamente).

Em seguida, analisaram-se as diferenças médias de desempenho dos alunos nas provas da bateria entre os clusters (alto, médio e baixo QI) para cada grupo etário (5, 7 e 9 anos). Para cada uma dessas análises foi gerada uma representação gráfica que ilustra tais diferenças de perfis. Assim, na Figura 1 apresentam-se os resultados para o grupo etário dos 5 anos.
Verifica-se que os alunos do Grupo 1 (QI alto) obtiveram uma pontuação média nas provas da bateria de 12,60 , sendo de registrar um desvio superior a três pontos na prova verbal de pensamento

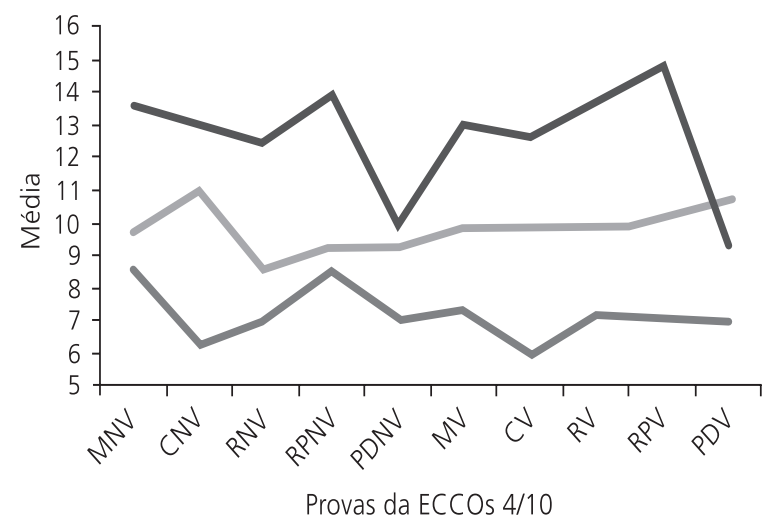

$$
\text { Grupo } 1-\text { Grupo } 2 \longrightarrow \text { Grupo } 3
$$

Figura 1. Resultados com os três clusters para os 5 anos. Porto, 2013. Nota: MNV: Memória Não-Verbal; CNV: Compreensão Não-Verbal; RNV: Raciocínio Não-Verbal; RPNV: Resolução de Problemas Não-Verbal; PDNV: Pensamento Divergente Não-Verbal; MV: Memória Verbal; CV: Compreensão Verbal; RV: Raciocínio Verbal; RPV: Resolução de Problemas Verbal; PDV: Pensamento Divergente Verbal; ECCOs 4/10: Escala de Competências Cognitivas para Crianças dos 4 aos 10 anos. 
Tabela 2

Caracterização dos três grupos de clusters, segundo níveis gerais de desempenho cognitivo. Porto, 2013

\begin{tabular}{|c|c|c|c|c|c|c|c|c|c|c|c|}
\hline Grupos & Valores & MNV & CNV & RNV & RPNV & PDNV & $\mathrm{MV}$ & CV & RV & RPV & PDV \\
\hline \multirow[t]{3}{*}{1} & $N$ & 39 & 39 & 39 & 39 & 39 & 39 & 39 & 39 & 39 & 39 \\
\hline & $M$ & 12,3 & 11,95 & 13,08 & 12,59 & 9,54 & 12,10 & 13,21 & 12,95 & 14,23 & 9,13 \\
\hline & $D P$ & 3,18 & 2,86 & 2,93 & 3,60 & 3,52 & 2,52 & 2,63 & 2,22 & 2,64 & 3,98 \\
\hline \multirow[t]{3}{*}{2} & $N$ & 131 & 131 & 131 & 131 & 131 & 131 & 131 & 131 & 131 & 131 \\
\hline & $M$ & 9,56 & 10,82 & 8,99 & 9,76 & 9,90 & 9,85 & 9,81 & 10,05 & 9,90 & 9,99 \\
\hline & $D P$ & 3,27 & 2,29 & 3,36 & 3,21 & 3,06 & 2,72 & 3,12 & 2,50 & 2,41 & 3,72 \\
\hline \multirow[t]{3}{*}{3} & $N$ & 61 & 61 & 61 & 61 & 61 & 61 & 61 & 61 & 61 & 61 \\
\hline & $M$ & 8,30 & 6,70 & 7,21 & 7,66 & 7,56 & 6,85 & 6,21 & 6,57 & 7,51 & 7,75 \\
\hline & $D P$ & 2,87 & 2,62 & 2,93 & 2,85 & 2,67 & 3,28 & 3,24 & 3,03 & 2,78 & 3,30 \\
\hline
\end{tabular}

Nota: MNV: Memória Não-Verbal; CNV: Compreensão Não-Verbal; RNV: Raciocínio Não-Verbal; RPNV: Resolução de Problemas Não-Verbal; PDNV: Pensamento Divergente Não-Verbal; MV: Memória Verbal; CV: Compreensão Verbal; RV: Raciocínio Verbal; RPV: Resolução de Problemas Verbal; PDV: Pensamento Divergente Verbal; N: Dimensão da amostra; M: Média; DP: Desvio-Padrão.

divergente $(M=9,29 ; \pm 5,09)$, cumprindo assim o critério de diferenciação cognitiva estipulado. Não atingindo o critério de diferenciação cognitiva, observam-se ainda no Grupo 1 oscilações importantes nas provas não-verbais de resolução de problemas $(M=13,86 ; \pm 4,22)$ e pensamento divergente $(M=10,00 ; \pm 2,65)$, e nas provas verbais de raciocínio $(M=13,71 ; \pm 2,98)$ e resolução de problemas $(M=14,71 ; \pm 2,50)$.

No Grupo 2 (QI médio), a média de desempenho obtida foi 9,79. O perfil cognitivo apresenta-se bastante homogêneo, sem oscilações tão expressivas nas diversas provas por comparação com o Grupo 1. No entanto, é percetível uma ligeira oscilação das provas não-verbais de compreensão $(M=10,90 ; \pm 1,99)$ e raciocínio $(M=8,51 ; \pm 3,99)$. Nesse grupo verifica-se uma maior oscilação das pontuações nas provas não-verbais.

Por fim, a média de desempenho cognitivo obtido pelos alunos do Grupo 3 (QI baixo) foi de 7,16 e, também nesse caso, nenhuma prova cognitiva cumpriu com o critério de diferenciação. No entanto, verifica-se que as provas não-verbais de memória $(M=8,47 ; \pm 2,09)$ e resolução de problemas $(M=8,42 ; \pm 2,22)$ e a prova verbal de compreensão $(M=6,00 ; \pm 3,06)$ foram as que mais oscilaram. Também nesse grupo verifica-se uma maior oscilação dos resultados nas provas não-verbais.

A partir da Figura 1 pode-se também constatar que há diferenças significativas entre os três grupos de desempenho cognitivo.
Verifica-se que o Grupo 1 se diferencia significativamente do Grupo 2 em todas as provas, exceto as de compreensão não-verbal $(F=36,74$; $p=0,06)$ e verbal $(F=13,03 ; p=0,12)$, e de pensamento divergente não-verbal $(F=3,45$; $p=0,85)$ e verbal $(F=7,81 ; p=0,60)$. O Grupo 1 apenas não se diferencia significativamente do Grupo 3 na prova de pensamento divergente em ambos os conteúdos, verbal e não-verbal $(F=7,81$; $p=0,29$ e $F=3,45 ; p=0,13$, respectivamente).

Finalmente, o Grupo 2 diferencia-se significativamente do Grupo 3 em todas as provas, excluindo as seguintes de conteúdo não-verbal: memória $(F=7,11 ; p=0,26)$, raciocínio $(F=5,02$; $p=0,32)$ e resolução de problemas $(F=9,76$; $p=0,52)$. A Figura 2 apresenta a representação gráfica das diferenças médias de desempenho cognitivo entre os três grupos para os 7 anos.

Obteve-se uma média de 12,19 para os resultados do Grupo 1, que apresenta um perfil cognitivo com bastantes oscilações, mas com nenhuma prova que cumpra o critério de três desvios da média. Porém, observaram-se oscilações acentuadas nas provas não-verbais de memória $(M=10,93 ; \pm 3,71)$, pensamento divergente $(M=10,97 ; \pm 4,20)$ e raciocínio $(M=13,21 ; \pm 3,31)$, bem como nas provas verbais de compreensão $(M=13,93 ; \pm 2,09)$, raciocínio $(M=13,50 ; \pm 2,03)$, resolução de problemas $(M=13,43 ; \pm 2,88)$ e pensamento divergente $(M=9,86 ; \pm 4,20)$. Dessa forma, o Grupo 1 apresenta uma maior oscilação do conteúdo verbal. 


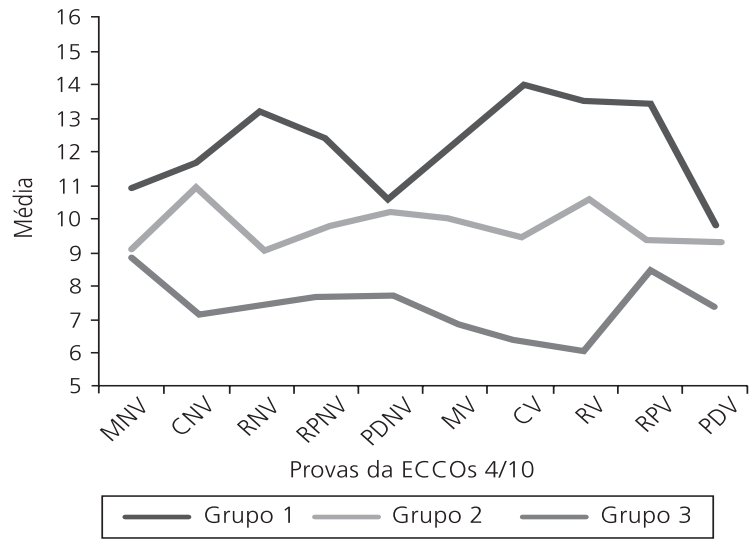

Figura 2. Resultados com os três clusters para os 7 anos. Porto, 2013. Nota: MNV: Memória Não-Verbal; CNV: Compreensão Não-Verbal; RNV: Raciocínio Não-Verbal; RPNV: Resolução de Problemas Não-Verbal; PDNV: Pensamento Divergente Não-Verbal; MV: Memória Verbal; CV: Compreensão Verbal; RV: Raciocínio Verbal; RPV: Resolução de Problemas Verbal; PDV: Pensamento Divergente Verbal; ECCOs 4/10: Escala de Competências Cognitivas para Crianças dos 4 aos 10 anos.

No Grupo 2, a média foi de 9,77, e, apesar dos desvios não serem superiores a 1,5 valores, foi possível verificar uma ligeira oscilação na prova não-verbal de compreensão $(M=10,98 ; \pm 2,32)$ e na prova verbal de raciocínio $(M=10,50 ; \pm 2,65)$.

Finalmente, o Grupo 3 atingiu uma média de 7,42, registrando-se alguma oscilação nos resultados obtidos. Essa oscilação ocorre na prova não-verbal de memória $(M=9,00 ; \pm 2,95)$ e nas provas verbais de compreensão $(M=6,35 ; \pm 3,30)$, raciocínio $(M=6,13 ; \pm 2,97)$ e resolução de problemas $(M=8,52 ; \pm 2,21)$. Novamente, nesse grupo verifica-se uma maior oscilação nas provas cognitivas de conteúdo verbal.

A Figura 2 também indica que os três grupos não se diferenciam significativamente entre si nas provas de memória não-verbal $(F=1,89 ; p=0,16)$ e de pensamento divergente verbal $(F=2,25$; $p=0,11$. Além disso, e prestando atenção às restantes provas cognitivas, verifica-se que o Grupo 1 não se diferencia significativamente do Grupo 2 nas provas não-verbais de compreensão $(F=22,25$; $p=0,59)$ e pensamento divergente $(F=5,84$; $p=0,90)$. O Grupo 1 diferencia-se do Grupo 3 em todas as provas. E, finalmente, o Grupo 2 não se diferencia do Grupo 3 nas provas de raciocínio não- problemas verbal $(F=17,72 ; p=0,46)$. A Figura 3 apresenta a representação gráfica para o grupo etário dos 9 anos.

O Grupo 1 obteve uma média de 11,79, e os resultados demostram diferenciação significativa nas provas de pensamento divergente, não-verbal $(M=8,56 ; \pm 3,13)$ e verbal $(M=8,50 ; \pm 3,43)$, com desvio superior a três pontos. Esse é um perfil cognitivo bastante heterogêneo. Também as provas de raciocínio não-verbal $(M=13,22 ; \pm 2,46)$, compreensão verbal $(M=12,89 ; \pm 2,32)$ e resolução de problemas verbal $(M=14,67 ; \pm 2,50)$ evidenciam oscilações consideráveis. Nesse grupo verifica-se uma maior oscilação das provas de conteúdo verbal.

O Grupo 2 alcançou uma média de 10,06, com desvios nunca superiores a um ponto, traduzindo-se num perfil bastante homogêneo. Finalmente, o Grupo 3 apresenta uma média de 7,07 e também um perfil cognitivo em que nenhuma prova se destacou. Todavia, foi possível observar uma ligeira oscilação das provas de pensamento divergente de conteúdo não-verbal $(M=8,00 ; \pm 2,24)$ e verbal $(M=9,05 ; \pm 2,12)$.

A partir da Figura 3 também se observam diferenças significativas entre os três grupos em todas as provas, com a exceção da do pensamento divergente verbal $(F=1,10 ; p=0,34)$. Assim, tendo em conta as restantes provas, verifica-se que o Grupo 1 não se diferencia significativamente do Grupo 3 na prova de pensamento divergente não-verbal ( $F=8,34 ; p=0,77)$, nem do Grupo 2 nas provas não-verbais de compreensão $(F=18,66$; $p=0,32)$ e resolução de problemas $(F=12,06$; $p=0,15)$, e na prova verbal de memória ( $F=13,94$; $p=0,12$ ). Por sua vez, o Grupo 2 diferencia-se do Grupo 3 em todas as provas. Apesar da superioridade do Grupo 1 nas várias provas, este apresenta um desempenho inferior aos Grupos 2 e 3 na prova de pensamento divergente verbal e uma prestação também inferior ao Grupo 2 na mesma prova de conteúdo não-verbal.

\section{Discussão}

A infância é um período em que ocorrem grandes mudanças no nível das habilidades cogni- 


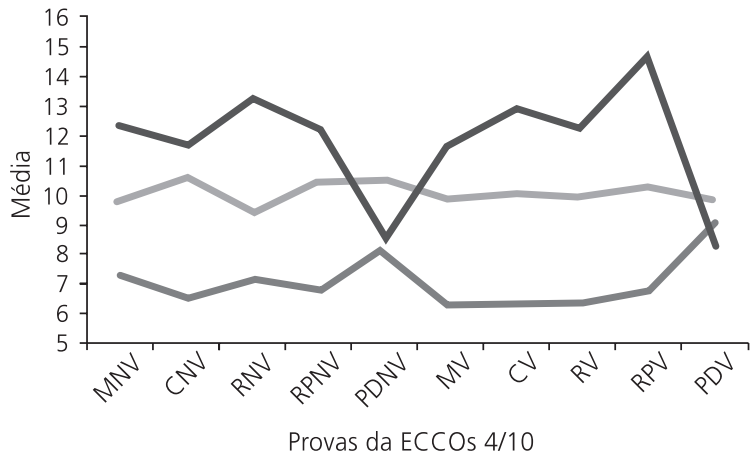

- Grupo $1-$ Grupo $2 \longrightarrow$ Grupo 3

Figura 3. Resultados com os três clusters para os 9 anos. Porto, 2013. Nota: MNV: Memória Não-Verbal; CNV: Compreensão Não-Verbal; RNV: Raciocínio Não-Verbal; RPNV: Resolução de Problemas Não-Verbal; PDNV: Pensamento Divergente Não-Verbal; MV: Memória Verbal; CV: Compreensão Verbal; RV: Raciocínio Verbal; RPV: Resolução de Problemas Verbal; PDV: Pensamento Divergente Verbal. ECCOs 4/10: Escala de Competências Cognitivas para Crianças dos 4 aos 10 anos.

tivas, sendo necessário conhecer as peculiaridades do desenvolvimento cognitivo nessa faixa etária para se promoverem medidas educativas que favoreçam esse desenvolvimento. Nesse sentido, o presente estudo pretendeu contribuir com novos dados empíricos para o debate em torno da diferenciação cognitiva na infância, particularmente sobre a sua verificação (ou não), em quais funções cognitivas e em quais idades.

Os resultados desta pesquisa, atendendo à estabilidade de $g$ observada nas faixas etárias consideradas (5, 7 e 9 anos), não confirmam a hipótese da diferenciação cognitiva, especialmente nos grupos de crianças com desempenho cognitivo médio e inferior (QI médio e QI baixo). Esses resultados corroboram a ausência de uma diferenciação cognitiva na infância, encontrada em outros estudos (Juan-Espinosa et al., 2006; Juan-Espinosa, García, Colom, \& Abad, 2000; Molenaar et al., 2010; Rietveld et al., 2003). No mesmo sentido, tem-se a posição de outros autores que associam o início da adolescência, fruto de experiências acadêmicas mais diferenciadas, a uma mudança progressiva na habilidade intelectual genérica ( $g$ ou Gf), formando-se habilidades cognitivas mais diferenciadas (Carlstedt, 2001; Facon, 2008; Kan et al., 2011; Kvist \& Gustafsson, 2008).
No entanto, se o conjunto dos resultados no presente estudo aponta para a não diferenciação das habilidades cognitivas, uma análise mais cuidadosa destaca alguma diferenciação suportada na heterogeneidade dos perfis cognitivos dos alunos de nível de desempenho mais elevado. Esse padrão mais diferenciado das crianças com QI elevado parece corroborar a teoria SLODR (Spearman, 1927), também replicada em estudos mais recentes (Coyle \& Rindermann, 2013; Reynolds \& Keith, 2007; Reynolds, Keith, \& Beretvas, 2010; Tucker-Drob, 2009). A razão dessa relação inversa tem tido várias explicações, principalmente uma melhor eficiência dos processos cognitivos centrais e do sistema de processamento de informação (Detterman, 1991, 1999) ou uma maior apropriação das experiências escolares (Abad, Colom, Juan-Espinosa, \& García, 2003) pelos indivíduos com QI elevado. Finalmente, e ao contrário de outros estudos (Brito, Almeida, Ferreira, \& Guisande, 2011; Johnson \& Bouchard, 2005), não se observou uma diferenciação cognitiva em função dos conteúdos das tarefas.

De qualquer modo, tomando o conjunto de provas verbais e não-verbais ao longo das três idades, verificou-se uma maior heterogeneidade de desempenho nas provas não-verbais em crianças de 5 anos, e uma maior variação nas provas verbais das crianças de 7 e 9 anos. De acordo com a teoria de investimento (Cattell, 1987), as diferenças de desempenho ocorrem em função das experiências, da motivação e dos padrões de interesse dos indivíduos. Procurando explicar essa diferença e pensando nos contextos educativos, pode-se assumir um predomínio das atividades manipulativas e figurativas no pré-escolar (criança de 5 anos), e um predomínio das atividades verbais no ensino fundamental (crianças de 7 e 9 anos).

Há ainda que se destacar que as crianças com melhor desempenho em provas que apelam ao raciocínio e à compreensão apresentam um desempenho mais baixo nas provas de pensamento divergente e, particularmente, de conteúdo verbal. Tomando as crianças mais velhas da amostra (9 anos), verifica-se que o grupo de QI elevado se destaca nas várias habilidades cognitivas, com exceção do pensamento divergente, enquanto os grupos de médio e baixo QI apresentam uma estabilidade nas 
médias. Destaca-se ainda que o grupo de baixo QI mostra um melhor desempenho nas provas de pensamento divergente (Figura 3). Esse dado pode significar um menor investimento, por parte da escola e dos alunos mais adaptados academicamente, nas tarefas relacionadas com a imaginação e a criatividade, dada a ênfase nas habilidades cognitivas associadas à compreensão, raciocínio e resolução de problemas.

A terminar, duas limitações importantes poderão ser apontadas a este estudo. A primeira diz respeito à amplitude de idades em causa. Estudos futuros deverão considerar faixas etárias com maior amplitude, para melhor se aferir sobre uma eventual alteração da estrutura cognitiva na infância. A segunda diz respeito ao reduzido número de sujeitos em cada grupo de desempenho cognitivo, o que coloca constrangimentos às análises diferenciais.

A par do interesse da questão para a investigação, a mesma pode ter implicações relevantes para a área educativa, principalmente quando se pensa que as opções curriculares e metodológicas dos professores no ensino fundamental favorecem certas habilidades cognitivas em prejuízo de outras. Pode-se questionar a sua ênfase nos conteúdos verbais e linguísticos em detrimento de tarefas mais práticas e perceptivo-espaciais, o mesmo ocorrendo em relação ao desenvolvimento do pensamento divergente das crianças, com a entrada na escolarização formal. Uma maior heterogeneidade das práticas de ensino e de aprendizagem poderá melhor responder à diversidade cognitiva e cultural dos alunos, o que é hoje amplamente valorizado no quadro de uma educação inclusiva e de qualidade para todos.

\section{Colaboradores}

Todos os autores contribuíram na concepção e desenho do estudo, análise de dados e redação final.

\section{Referências}

Abad, F. J., Colom, R., Juan-Espinosa, M., \& García, L. F. (2003). Intelligence differentiation in adult samples. Intelligence, 31(2), 157-166. http://dx.doi.org/10.
Almeida, L. S. (2002). As aptidões na definição e avaliação da inteligência: o concurso da análise fatorial. Paidéia, 12(23), 5-17. http://dx.doi.org/10.1590/S0103-863X 2002000200002

Bergman, L. R. (1998). A pattern-oriented approach to studying individual development: Snapshots and processes. In R. B. Cairns, L. R. Bergman, \& J. Kagan (Eds.), Methods and models for studying the individual (pp.83-122). Thousand Oaks: Sage.

Brito, L., \& Almeida, L. S. (2009). Escala de competências cognitivas para crianças - ECCOs 4/10: manual. Porto: Edição dos autores.

Brito, L., Almeida, L. S., Ferreira, A., \& Guisande, M. (2011). Contribución de los procesos y contenidos a la diferenciación cognitiva en la infancia: Un estudio con escolares portugueses. Infancia y Aprendizaje, 34(3), 323-336. http://dx.doi.org/10.1174/021037 011797238540

Carlstedt, B. (2001). Differentiation of cognitive abilities as a function of level of general intelligence: A latent variable approach. Multivariate Behavioral Research, 36(4), 589-609. http://dx.doi.org/10.1207/S1532790 6MBR3604

Cattell, R. B. (1987). Intelligence: Its structure, growth, and action. Amsterdam: North-Holland.

Coyle, T. R., \& Rindermann, H. (2013). Spearman's law of diminishing returns and national ability. Personality and Individual Differences, 55(4), 406-410. http://dx. doi.org/10.1016/j.paid.2013.03.023

Detterman, D. K. (1991). Reply to Deary and Pagliari: Is $g$ intelligence or stupidity? Intelligence, 15(2), 251-255. http://dx.doi.org/10.1016/0160-2896(91)90034-B

Detterman, D. K. (1999). The psychology of mental retardation. International Review of Psychiatry, 11(1), 26-33. http://dx.doi.org/10.1080/09540269974492

Everitt, B. S., Landau, S., \& Leese, M. (2001). Cluster analysis (4th ed.). London: Arnold Publishers.

Facon, B. (2004). Are correlations between cognitive abilities highest in low-IQ groups during childhood? Intelligence, 32(4), 391-401. http://dx.doi.org/10.10 16/j.intell.2004.06.002

Facon, B. (2006). Does age moderate the effect of IQ on the differentiation of cognitive abilities during childhood? Intelligence, 34(6), 375-386. http://dx.doi. org/10.1016/j.intell.2005.12.003

Facon, B. (2008). How does the strength of the relationships between cognitive abilities evolve over the life span for low-IQ vs high-IQ adults? Intelligence, 36(4), 339-349. http://dx.doi.org/10.1016/j.intell. 2007.11.004

Jensen, A. R. (2003). Regularities in Spearman's law of diminishing returns. Intelligence, 31(2), 95-105. http:// dx.doi.org/10.1016/S0160-2896(01)00094-0 
Johnson, W., \& Bouchard, T. J. (2005). The structure of human intelligence: It is verbal, perceptual, and image rotation (VPR), not fluid and crystallized. Intelligence, 33(4), 393-416. http://dx.doi.org/10.1016/j.intell. 2004.12.002

Juan-Espinosa, M., Cuevas, L., Escorial, S., \& García, L. F. (2006). Testing the indifferentiation hypothesis during childhood, adolescence, and adulthood. The Journal of Genetic Psychology, 167(1), 5-15. http://dx.doi.org/ 10.3200/GNTP.167.1.5-15

Juan-Espinosa, M., García, L. F., Colom, R., \& Abad, F. J. (2000). Testing the age related differentiation hypothesis through the Wechsler's scales. Personality and Individual Differences, 29(6), 1069-1075. http:// dx.doi.org/10.1016/S0191-8869(99)00254-8

Juan-Espinosa, M., García, L. F., Escorial, S., Rebollo, I., Colom, R., \& Abad, F. J. (2002). Age dedifferentiation hypothesis: Evidence from the WAIS III. Intelligence, 30(5), 395-408. http://dx.doi.org/10.1016/S0160-2 896(02)00092-2

Kan, K. J., Kievit, R. A., Dolan, C. V., \& van der Maas, H. L. J. (2011). On the interpretation of the $\mathrm{CHC}$ factor Gc. Intelligence, 39(5), 292-302. http://dx.doi.org/10. 1016/j.intell.2011.05.003

Kvist, A., \& Gustafsson, J. E. (2008). The relation between fluid intelligence and the general factor as a function of cultural background: A test of Cattell's investment theory. Intelligence, 36(5), 422-436. http://dx.doi.org/ 10.1016/j.intell.2007.08.004

Molenaar, D., Dolan, C. V., Wicherts, J. M., \& van der Maas, H. L. J. (2010). Modeling differentiation of cognitive abilities within the higher-order factor model using moderated factor analysis. Intelligence, 38(6), 611-624. http://dx.doi.org/10.1016/j.intell.2010.09. 002

Primi, R. (2002). Inteligência fluida: definição fatorial, cognitiva e neuropsicológica. Paidéia, 12(23), 57-75. http://dx.doi.org/10.1590/S0103-863X200200020 0005
Primi, R., Ferrão, M. E., \& Almeida, L. S. (2010). Fluid intelligence as a predictor of learning: A longitudinal multilevel approach applied to math. Learning and Individual Differences, 20(5), 446-451. http://dx.doi. org/10.1016/j.lindif.2010.05.001

Reynolds, M. R., \& Keith, T. Z. (2007). Spearman's law of diminishing returns in hierarchical models of intelligence for children and adolescents. Intelligence, 35(3), 267-281. http://dx.doi.org/10.1016/j.intell. 2006.08.002

Reynolds, M. R., Keith, T. Z., \& Beretvas, S. N. (2010). Use of factor mixture modeling to capture Spearman's law of diminishing returns. Intelligence, 38(2), 231241. http://dx.doi.org/10.1016/j.intell.2010.01.002

Rietveld, M. J. H., Dolan, C. V., van Baal, G. C. M., \& Boomsma, D. I. (2003). A twin study of differentiation of cognitive abilities in childhood. Behavior Genetics, 33(4), 367-381. http://dx.doi.org/10.1023/A:1025 388908177

Spearman, C. (1927). The abilities of man. New York: MacMillan.

Tideman, E., \& Gustafsson, J. E. (2004). Age-related differentiation of cognitive abilities in ages 3-7. Personality and Individual Differences, 36(8), 1965-1974. http://dx.doi.org/10.1016/j.paid.2003. 09.004

Tucker-Drob, E. M. (2009). Differentiation of cognitive abilities across the lifespan. Developmental Psychology, 45(4), 1097-1118. http://dx.doi.org/10.1037/a00 15864

Tusing, M. E., \& Ford, L. (2004). Examining preschool cognitive abilities using a CHC framework. International Journal of Testing, 4(2), 91-114. http://dx.doi.org/10. 1207/s15327574ijt0402_1

Recebido: agosto 12, 2014

Versão final: outubro 22, 2015

Aprovado: dezembro 3, 2015 
